\title{
Single Gold-nanoparticle-enhanced Raman Scattering of Individual Single-walled Carbon Nanotubes via Atomic Force Microscope Manipulation
}

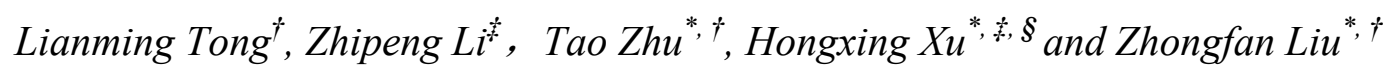

Center for Nanoscale Science and Technology, Beijing National Laboratory for Molecular Sciences,

State Key Laboratory for Structural Chemistry of Unstable and Stable Species, College of Chemistry and Molecular Engineering, Peking University, Beijing, 100871, China, State Key Laboratory for Surface Physics, Institute of Physics, Chinese Academy of Sciences, Beijing, 100080, China and

Division of Solid State Physics, Department of Physics, Lund University, Box 118, SE-22100, Lund,

Sweden

${ }^{*}$ Corresponding authors. zhutao@pku.edu.cn, $\underline{\text { hxxu@aphy.iphy.ac.cn, zfliu@pku.edu.cn }}$

${ }^{\dagger}$ Peking University

$\ddagger$ Chinese Academy of Sciences

$\S$ Lund University 


\section{How to estimate of the distance $D$ between a GNP and a SWNT?}

The AFM images are displayed in the contour mode. Although the convolution effect of the AFM tip makes the apparent lateral sizes of GNPs and SWNTs larger than their real sizes, the maximum heights and the corresponding top positions can be exactly distinguished, respectively. In Figure S1, the black and white rings represent the projection of a GNP, and the gray band under the GNP is the AFM image of the SWNT. The center line of the gray band, highlighted by the solid line, is regarded as the real position of the SWNT. The measured distances $(D)$ from the center of the GNP's projection in the AFM image to the SWNT are the distance between the GNP and the SWNT, which are $\sim 15 \mathrm{~nm}, \sim 40 \mathrm{~nm}$, $\sim 60 \mathrm{~nm}$ and $200 \mathrm{~nm}$, respectively. The derivation is estimated less than $\pm 3 \mathrm{~nm}$ based on the error in determining the position of the center of a GNP's projection.
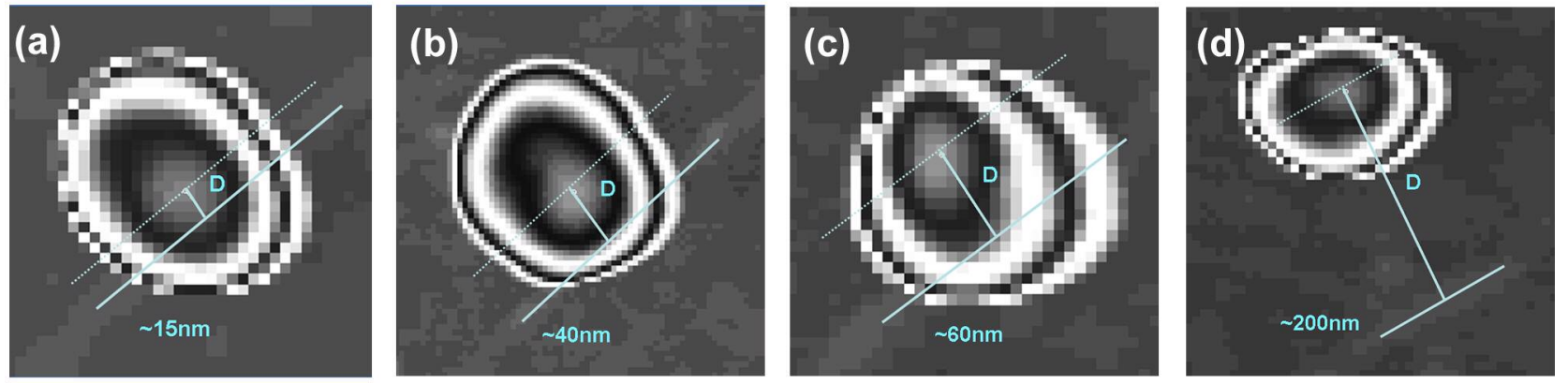

Figure S1. AFM images in contour mode, showing the distance between the center of the GNP's projection and the SWNT after a series of AFM manipulation. The image sizes are $246 \times 246 \mathrm{~nm}$ for (a), (b) and (c), and $345 \times 345 \mathrm{~nm}$ for (d).

\section{Does the GNP contact the SWNT?}

The scheme in Figure S2 is used to evaluate the possibility of the contact between the GNP with radius $R$ and the SWNT with diameter $d_{t}$, where the GNP is assumed to be spherical. When the GNP and the SWNT contact each other on a flat surface, the critical distance $\left(D_{c}\right)$ between the central 
positions of the GNP's projection and the SWNT can be estimated according to the Pythagorean Theorem. For the GNP displayed in Figure S1 (Figure 3 in the main text), the diameter is $80 \mathrm{~nm}$. When it contacts the SWNT of $0.9 \mathrm{~nm}$ in diameter, the critical distance $\left(D_{c}\right)$ is calculated to be $8.5 \mathrm{~nm}$. For the smallest distance $(D \sim 15 \mathrm{~nm})$ used in Figure 3, the GNP unlikely contacts the SWNT, neither does for the larger distances. While in Figure 2.a(III), the distance is estimated to be $\sim 10 \mathrm{~nm}$, but the critical distance is calculated to be $21 \mathrm{~nm}$ for the GNP $(R=55 \mathrm{~nm})$ and the SWNT $\left(d_{t}=4.2 \mathrm{~nm}\right)$. Hence the GNP in Figure 2.a(III) likely contacts the SWNT.

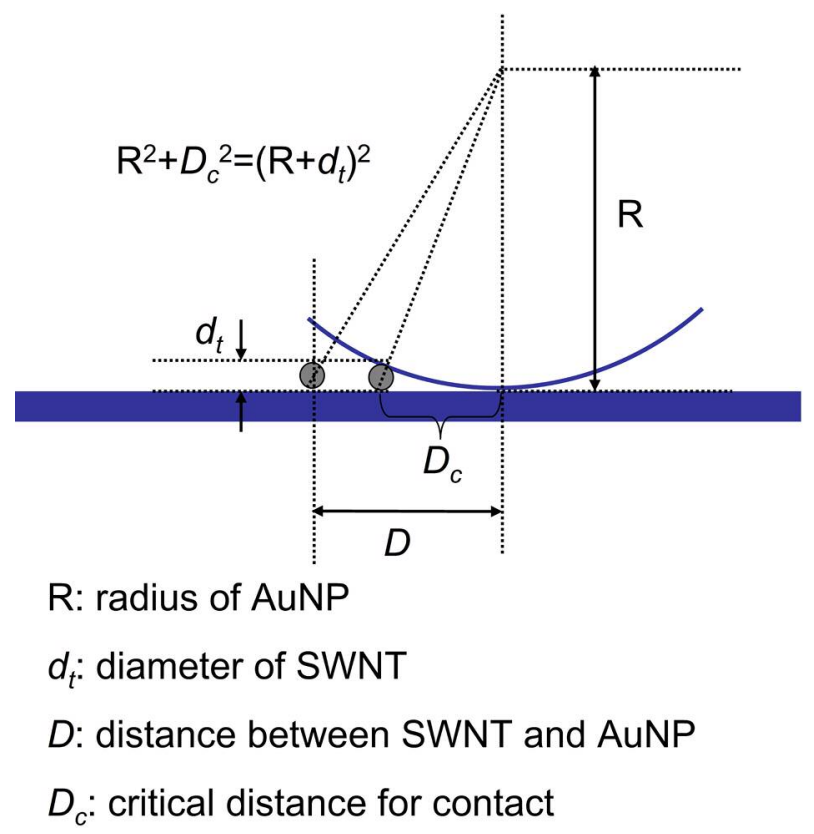

Figure S2. The schematic view of the GNP and the SWNT to estimate the critical distance for contact

\section{Supplemental evidence for the confirmation of the SWNT bundle in Figure 2}

The Raman spectra in Figure 2(c) in the main text show that a weak peak at $173 \mathrm{~cm}^{-1}$ appeared after the GNP was pushed closer to the SWNT. Since the signal/noise ratio is relatively weak, we show the anti-stocks region of RBM in Figure S3, where a weak peak at $-173 \mathrm{~cm}^{-1}$ also appears, to convince that it is reliably from another SWNT in this bundle instead of noise signal. 


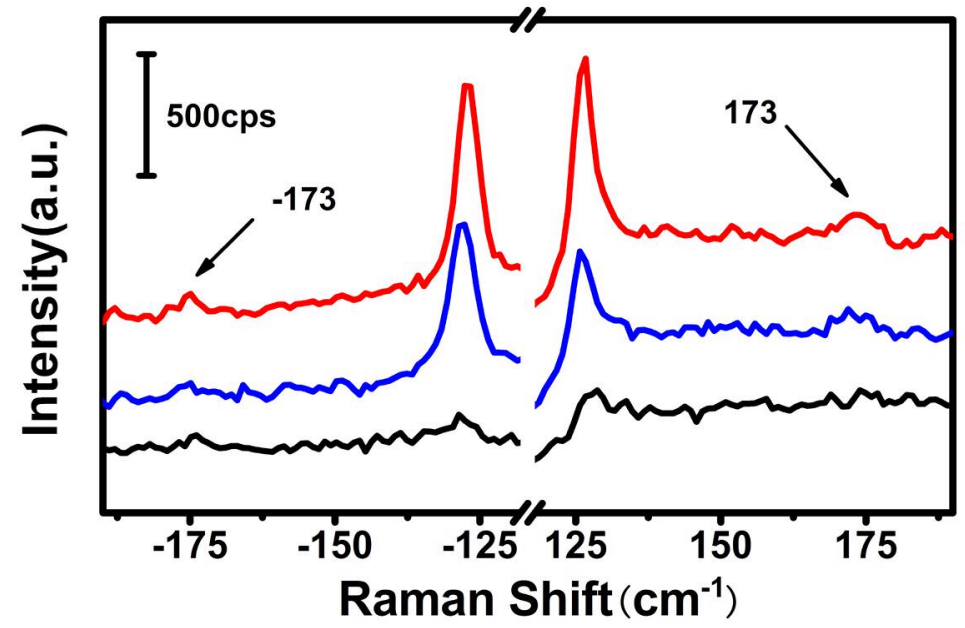

Figure S3. Anti-stokes and Stokes Raman spectra (RBM region) of the SWNT shown in Figure 2(a) in the main text.

\section{The evidence for SERS only caused by the arrow-indicated GNP in Figure 3}

To confirm the Raman enhancement of the SWNT in Figure 3 in the main text is caused only by the arrow-indicated GNP instead of the nearby GNPs, we measured the Raman intensity and the polarization dependence of the same section of the SWNT before and after the other nearby GNPs being pushed away and other sections of the same SWNT without GNPs. Figure S4 shows the corresponding AFM images and the polarization dependence of the corresponding Raman intensities ( $\mathrm{G}^{+}$band). Moving other nearby GNPs away do not change the Raman intensity and peak position of the SWNT, as shown in Figure S4(c) and Table S1. So the nearby GNPs do not contribute to the Raman enhancement of the SWNT and the Raman enhancement in Figure 3g in the main text is caused only by the arrow-indicated GNP. On the other hand, it is also indicated that there is no observable damage caused by laser irradiation or the bake process since there is no observable change in the Raman spectra before and after this manipulation. 

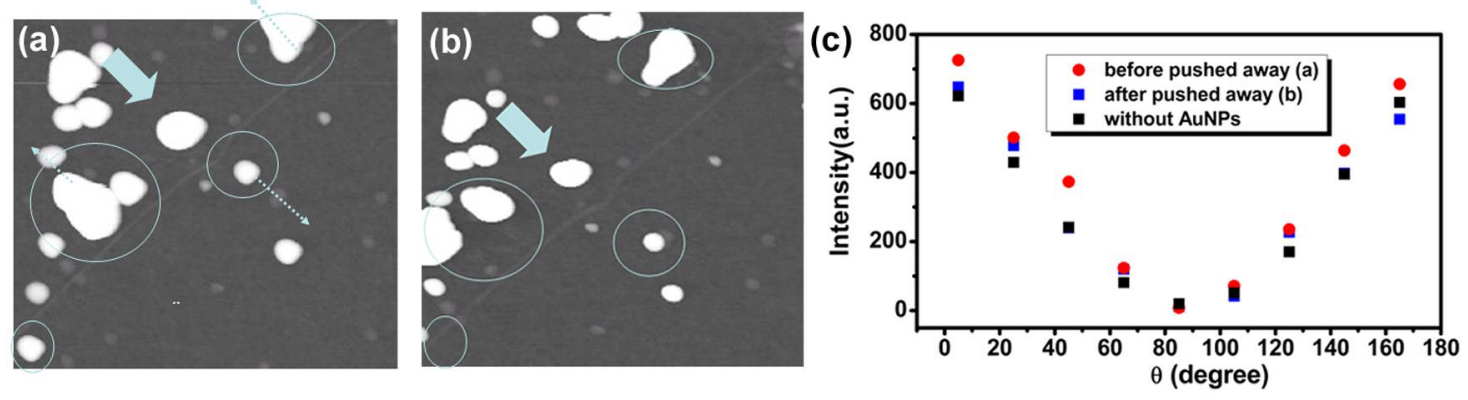

Figure S4. (a), (b) AFM images $(1.8 \times 1.8 \mu \mathrm{m})$ of the same area before and after other nearby GNPs are pushed away. (c) Raman intensities and the polarization dependence of the sections of SWNT shown in (a), (b) and that without GNPs.

Table S1. $\mathrm{G}^{+}$peak positions $\left(\omega\right.$, in $\left.\mathrm{cm}^{-1}\right)$ of the sections of the SWNT shown in Figure S4(a), (b) and that without GNPs at different polarization angles

\begin{tabular}{|c|c|c|c|}
\hline \multirow{2}{*}{ Angle/degree } & \multicolumn{3}{|c|}{$\omega\left(\mathrm{G}^{+}\right)$} \\
\cline { 2 - 4 } & without GNPs & in Figure S4(a) & in Figure S4(b) \\
\hline 5 & 1589.6 & 1589.8 & 1589.9 \\
\hline 25 & 1589.7 & 1589.7 & 1589.9 \\
\hline 45 & 1589.8 & 1589.9 & 1589.9 \\
\hline 65 & 1589.9 & 1589.6 & 1589.6 \\
\hline 85 & $/$ & $/$ & $/$ \\
\hline 105 & 1589.7 & 1589.6 & 1589.7 \\
\hline 125 & 1589.6 & 1589.6 & 1589.9 \\
\hline 145 & 1590.0 & 1589.8 & 1590.0 \\
\hline 165 & 1589.6 & 1589.9 & 1589.9 \\
\hline
\end{tabular}

